\title{
SOME DIFFERENTIAL GEOMETRIC PROPERTIES OF CODIMENSION-ONE FOLIATIONS OF POLYNOMIAL GROWTH
}

\author{
GEN-ICHI OSHIKIRI
}

(Received July 3, 2000, revised January 16, 2001)

\begin{abstract}
We show that a codimension-one minimal foliation with growth at most 2 of a complete Riemannian manifold with non-negative Ricci curvature is totally geodesic. We present some foliated versions of the result given by Alencar and do Carmo, and of minimal graphs by Miranda. Further, we simplify the proof of Meeks' result concerning constant mean curvature foliations of 3-dimensional Euclidean space.
\end{abstract}

1. Introduction. In $[\mathrm{Me}]$, Meeks proved that any codimension-one constant mean curvature foliation on the three dimensional Euclidean space is totally geodesic. In the case when the ambient manifold is compact, there have been known many results stating that minimal or constant mean curvature foliations are totally geodesic (see, e.g., [BKO], [O1], [Rh], [Td]). However, if the ambient space is non-compact, then there are only a few results asserting this phenomina (cf. [Sol]). In this paper, we shall study this problem under some additional assumptions. We first prove the following.

THEOREM 1. Let $(M, g, \mathcal{F})$ be a codimension-one minimal foliation of a complete Riemannian manifold with non-negative Ricci curvature. If the growth $\operatorname{gr}(L)$ of a leaf $L \in \mathcal{F}$ is not greater than 2, then $L$ is totally geodesic. In particular, if $\operatorname{gr}(\mathcal{F}) \leq 2$, then $\mathcal{F}$ is totally geodesic, and $(M, g)$ is locally a Riemannian product of a leaf of $\mathcal{F}$ and an orbit of $N$, where $N$ is a unit vector field on $M$ orthogonal to $\mathcal{F}$.

In Theorem 1, we have the same conclusion, if we replace the assumption " $(M, g)$ being with non-negative Ricci curvature" by weaker one " $\operatorname{Ric}(N, N) \geq 0$ ".

Note that, Theorem 1 does not hold if we simply drop the growth condition. In fact, from the counter examples to the Bernstein problem [BoDG], we can construct codimension-one minimal foliations of the Euclidean space $R^{n+1}$, for $n \geq 8$, by translating minimal graphs. In these examples, the growth of leaves are known to be $n$ (see also [GT]). Thus, the growth condition in Theorem 1 could be weakend until $\operatorname{gr}(\mathcal{F}) \leq 7$. But, this is an open problem.

Next, relating to the growth condition of $\mathcal{F}$, we prove

THEOREM 2. Let $(M, g)$ be a connected oriented complete Riemannian manifold, and $\mathcal{F}$ be a transversely oriented codimension-one foliation on $M$. If a leaf $L \in \mathcal{F}$ is of polynomial

2000 Mathematical Subject Classification. 53C12. 
growth, then it holds that

$$
\inf _{x \in L}\left\{\operatorname{Ric}(N, N)(x)+|A|^{2}(x)+N(h)(x)\right\} \leq 0,
$$

where $N$ is a unit vector field on $M$ orthogonal to $\mathcal{F}$, Ric is the Ricci curvature of $(M, g),|A|^{2}$ is the square of the norm of the second fundamental form of $\mathcal{F}$, and $h$ is the mean curvature function of $\mathcal{F}$ (see Section 2 for the definitions).

This result is, as is explained in Section 3, related to that of Alencar and do Carmo [AdC1]. Using a similar technique, we can obtain a foliated version of Miranda's result on minimal graphs ([Mi]).

THEOREM 3. Let $\mathcal{F}$ be a codimension-one minimal foliation of the Euclidean space $R^{n+1}$. If the growth of $L \in \mathcal{F}$ is at most $k \in N$, then

$$
\limsup _{r \rightarrow \infty} \frac{\int_{B(r)}|A|^{2}}{r^{k-2}}<\infty .
$$

These results and some corollaries are proved in Section 3.

Finally, in Section 4, we give a simple proof of the Meeks' result mentioned at the beginning of this section.

The author would like to thank the referees for many useful suggestions, which have considerably improved the presentation of the paper.

2. Preliminaries. Let $(M, g, \mathcal{F})$ be a codimension-one foliation of a complete connected Riemannian manifold $(M, g)$. As the results of this paper are valid when we lift everything onto a finite covering space of $M$, we may assume that $M$ and $\mathcal{F}$ are oriented without loss of generality. Hence we can choose a unit vector field $N$ on $M$ perpendicular to $\mathcal{F}$ everywhere on $M$ so that the orientation of $M$ coincides with the one given by $\mathcal{F}$ and $N$. Note that, as the dimension of the orthogonal complement $\mathcal{F}^{\perp}$ to $\mathcal{F}$ is one, $\mathcal{F}^{\perp}$ is always integrable and the leaves of $\mathcal{F}^{\perp}$ are the orbits of $N$. For simplicity of our calculation, we set $\operatorname{dim}(M)=n+1$. Thus $\operatorname{dim}(\mathcal{F})=n$. Denote by $\nabla$ the Riemannian connection of $(M, g)$. The curvature tensor $R$ of $(M, g)$ is defined by

$$
R(X, Y) Z=\nabla_{X} \nabla_{Y} Z-\nabla_{Y} \nabla_{X} Z-\nabla_{[X, Y]} Z
$$

for $X, Y, Z \in T M$. Let $\left\{V_{1}, V_{2}, \ldots, V_{n+1}\right\}$ be a local orthonormal frame of $T M$. The Ricci curvature tensor $\operatorname{Ric}(X, Y)$ of $(M, g)$ is defined by

$$
\operatorname{Ric}(X, Y)=\sum_{i=1}^{n+1}\left\langle R\left(V_{i}, X\right) Y, V_{i}\right\rangle
$$

for $X, Y \in T M$. Here $\langle X, Y\rangle$ means $g(X, Y)$. A Riemannian manifold $(M, g)$ satisfying $\operatorname{Ric}(X, Y) \geq 0$ for all $X, Y \in T M$ is said to be with non-negative Ricci curvature. 
Let $L$ be a leaf of a foliation $\mathcal{F}$. Choose a point $x \in L$ and define $B(r)=\{y \in$ $\left.L \mid d_{L}(x, y) \leq r\right\}$ for $r>0$. Here $d_{L}(\cdot, \cdot)$ means the distance function on $L$ induced by the Riemannian metric $\left.g\right|_{L}$ of $L$ from $(M, g)$. A leaf $L$ is said to be of polynomial growth if there are a positive integer $n$ and positive constants $a$ and $b$ so that $\operatorname{vol}(B(r)) \leq a r^{n}+b$ for all $r>0$. In this case, we write $\operatorname{gr}(L) \leq n$. If $\operatorname{gr}(L) \leq n$ for all $L \in \mathcal{F}$, then we write $\operatorname{gr}(\mathcal{F}) \leq n$.

Let $\left\{E_{1}, E_{2}, \ldots, E_{n}\right\}$ be a local orthonormal frame of $\mathcal{F}$. The mean curvature function $h$ of $\mathcal{F}$ is defined by

$$
h=\left\langle\sum_{i=1}^{n} \nabla_{E_{i}} E_{i}, N\right\rangle .
$$

Set $|A|^{2}=\sum_{i, j=1}^{n}\left\langle\nabla_{E_{i}} N, E_{j}\right\rangle^{2}$. Note that $|A|^{2}$ on a leaf $L \in \mathcal{F}$ is the square norm of the second fundamental form of $L$. As the vector field $\nabla_{N} N$ is everywhere tangent to $\mathcal{F}$, we define, on each leaf $L \in \mathcal{F}$, the one-form $\theta$ dual to $\nabla_{N} N$ by $\theta(E)=\left\langle\nabla_{N} N, E\right\rangle$ for $E \in T L$. By summing up $\left\langle R\left(N, E_{i}\right) E_{i}, N\right\rangle$ for $i=1,2, \ldots, n$ and noticing that $\sum_{i, j=1}^{n}\left\langle\nabla_{E_{i}} N, E_{j}\right\rangle\left\langle E_{i}, \nabla_{N} E_{j}\right\rangle=0$, since $\left\langle\nabla_{E_{i}} N, E_{j}\right\rangle$ being symmetric in $i$ and $j$, but $\left\langle E_{i}, \nabla_{N} E_{j}\right\rangle$ being skew-symmetric in $i$ and $j$, we have the following (see [O2] for a proof, cf. [BKO], [O1] and [Ra]).

PROPOSITION 1. Under the above notations, we have

$$
\operatorname{Ric}(N, N)+|A|^{2}+N(h)+|\theta|^{2}=\operatorname{div}_{L}\left(\nabla_{N} N\right),
$$

where $\operatorname{div}_{L}\left(\nabla_{N} N\right)$ is the divergence of the vector field $\nabla_{N} N$ on $L$ with respect to $\left(L,\left.g\right|_{L}\right)$.

We say that a codimension-one foliation $\mathcal{F}$ is a constant mean curvature foliation if the mean curvature function $h$ of $\mathcal{F}$ is constant on each leaf of $\mathcal{F}$. If $h \equiv 0$ on $M$, then each leaf of $\mathcal{F}$ is a minimal hypersurface of $(M, g)$, and $\mathcal{F}$ is called a minimal foliation. If $|A|^{2} \equiv 0$ on $M$, then each leaf of $\mathcal{F}$ is totally geodesic, and $\mathcal{F}$ is called a totally geodesic foliation.

PROPOSITION 2. Let $\mathcal{F}$ be a codimension-one constant mean curvature foliation with the mean curvature function $h$. Denote by $d_{L}$ the differentiation along a leaf $L \in \mathcal{F}$. Then it holds that $d_{L} N(h)=N(h) \theta$. In particular, if $N(h) \neq 0$ at a point $x \in L$, then $N(h)>0$ or $N(h)<0$ everywhere on $L$. Furthermore, on the saturated set where $N(h) \neq 0$, the vector field $N / N(h)$ preserves $\mathcal{F}$.

Proof. To prove $d_{L} N(h)=N(h) \theta$, we have only to show that $E N(h)=N(h) \theta(E)$ for $E \in T L$. Note that $E(h)=0$ for $E \in T L$. Thus, it follows that $E N(h)=E N(h)-$ $N E(h)=[E, N](h)=\langle[E, N], N\rangle N(h)=N(h) \theta(E)$. In particular, if $N(h)=0$ at $x \in L$, then, on any curve $c(t) \subset L$ starting from $x=c(0)$, we have an ordinary differential equation of the first order

$$
\frac{d N(h)}{d t}(c(t))=N(h)(c(t)) \theta\left(c^{\prime}(t)\right) .
$$

It is well-known that if $N(h)(c(0))=0$, then $N(h)(c(t)) \equiv 0$. 
For the last statement, we have only to prove $\langle[E, N / N(h)], N\rangle=0$ for $E \in \mathcal{F}$ (cf. [O3]). Indeed, this follows from the fact that $\langle[E, N / N(h)], N\rangle=\left\langle\nabla_{E}(N / N(h)), N\right\rangle-$ $\left\langle\nabla_{N / N(h)} E, N\right\rangle=E(1 / N(h))+\theta(E) / N(h)=(-E N(h)+\theta(E) N(h)) / N(h)^{2}=0$.

A leaf $L$ of a constant mean curvature foliation $\mathcal{F}$ with $\left.h\right|_{L} \neq 0$ is said to be stable if

$$
V_{f}^{\prime \prime}(0)=\int_{L}\left\{|d f|^{2}-f^{2}\left(\operatorname{Ric}(N, N)+|A|^{2}\right)\right\} \geq 0,
$$

where $f$ is any function having compact support on $L$ with $\int_{L} f=0$ (see [BdC], [BdCE]). In this paper, we omit the volume elements in integrations, because no confusion will be made. For a minimal leaf $L$, the definition of the stability of $L$ is given by the same inequality as above without the condition $\int_{L} f=0$ on $f$ (cf. [L], [O2]).

Proposition 3. Let $(M, g, \mathcal{F})$ be a codimension-one constant mean curvature foliation of a complete Riemannian manifold. If $N(h) \geq 0$ on $L \in \mathcal{F}$, then $L$ is stable. In particular, every leaf of a codimension-one minimal foliation is stable.

PROOF. By Proposition 1, it follows that

$$
\begin{aligned}
f^{2}\left(\operatorname{Ric}(N, N)+|A|^{2}\right) & =f^{2} \operatorname{div}_{L}\left(\nabla_{N} N\right)-f^{2}|\theta|^{2}-f^{2} N(h) \\
& =-\nabla_{N} N\left(f^{2}\right)-f^{2}|\theta|^{2}-f^{2} N(h)+\operatorname{div}_{L}\left(f^{2} \nabla_{N} N\right) .
\end{aligned}
$$

Thus, we have

$$
\begin{aligned}
V_{f}^{\prime \prime}(0) & =\int_{L}\left\{|d f|^{2}+2 f \nabla_{N} N(f)+f^{2}|\theta|^{2}+f^{2} N(h)\right\} \\
& =\int_{L}|d f+f \theta|^{2}+\int_{L} f^{2} N(h) .
\end{aligned}
$$

If $N(h) \geq 0$ on $L$, then $V_{f}^{\prime \prime}(0) \geq 0$, that is, $L$ is stable.

Note that the condition $\int_{L} f=0$ is not used here. Thus, if $\mathcal{F}$ is minimal, then, as $N(h) \equiv 0$, it follows that $V_{f}^{\prime \prime}(0)=\int_{L}|d f+f \theta|^{2} \geq 0$, that is, $L$ is stable.

\section{Proofs and corollaries.}

Proof of TheOREM 1. Let $L$ be a leaf of $\mathcal{F}$. By assumption, Proposition 1 becomes

$$
\operatorname{Ric}(N, N)+|A|^{2}+|\theta|^{2}=\operatorname{div}_{L}\left(\nabla_{N} N\right) .
$$

If $L$ is compact, by integrating this formula over $L$, we have

$$
\int_{L}\left\{\operatorname{Ric}(N, N)+|A|^{2}+|\theta|^{2}\right\}=0 .
$$

As each term of the integrand is non-negative, it follows that $|A|^{2}=0$, and hence $L$ is totally geodesic.

Now assume that $L$ is a non-compact leaf with the growth $\operatorname{gr}(L) \leq 2$. Fix $x \in L$. Then, by definition,

$$
\operatorname{vol}(B(r)) \leq a r^{2}+b \quad(r \geq 0),
$$


for some positive constants $a$ and $b$, where $B(r)=\left\{y \in L \mid d_{L}(x, y) \leq r\right\}$. Set $f(r)=$ $\int_{B(r)}|\theta|^{2}$ and $V(r)=\operatorname{vol}(B(r))$. It is known that $f(r)$ and $V(r)$ are locally Lipschitz, and thus a.e. differentiable (cf. $[\mathrm{CY}])$. By integrating the equation

$$
\operatorname{Ric}(N, N)+|A|^{2}+|\theta|^{2}=\operatorname{div}_{L}\left(\nabla_{N} N\right)
$$

over $B(r)$, we obtain

$$
\int_{B(r)}\left\{\operatorname{Ric}(N, N)+|A|^{2}+|\theta|^{2}\right\}=\int_{B(r)} \operatorname{div}_{L}\left(\nabla_{N} N\right)=\int_{\partial B(r)} \theta(v),
$$

where $v$ is the outward unit normal vector to $\partial B(r)$. As $\operatorname{Ric}(N, N) \geq 0$ and $|A|^{2} \geq 0$, we have

$$
\int_{B(r)}|\theta|^{2} \leq \int_{\partial B(r)}|\theta| \leq \sqrt{\int_{\partial B(r)} 1} \sqrt{\int_{\partial B(r)}|\theta|^{2}} .
$$

It follows that

$$
f(r)^{2} \leq f^{\prime}(r) V^{\prime}(r)
$$

because $f^{\prime}(r)=\int_{\partial B(r)}|\theta|^{2}$ and $V^{\prime}(r)=\int_{\partial B(r)} 1$.

Assume $\theta(x) \neq 0$. Then $f(r)>0$ for $r>0$. As $V^{\prime}(r)>0$, we have

$$
\frac{1}{V^{\prime}(r)} \leq \frac{f^{\prime}(r)}{f(r)^{2}}=\left(-\frac{1}{f(r)}\right)^{\prime} \text {. }
$$

Integrating this on $[r, R]$ with $0<r<R$, we get

$$
\int_{r}^{R} \frac{1}{V^{\prime}(r)} d r \leq \frac{1}{f(r)}-\frac{1}{f(R)} .
$$

The inequality

$$
\left(\int_{r}^{R} d r\right)^{2}=\left(\int_{r}^{R} \sqrt{V^{\prime}(r)} \sqrt{\frac{1}{V^{\prime}(r)}} d r\right)^{2} \leq\left(\int_{r}^{R} V^{\prime}(r) d r\right)\left(\int_{r}^{R} \frac{1}{V^{\prime}(r)} d r\right)
$$

implies

$$
\frac{(R-r)^{2}}{V(R)-V(r)} \leq \int_{r}^{R} \frac{1}{V^{\prime}(r)} d r .
$$

It follows that

$$
\frac{(R-r)^{2}}{V(R)-V(r)} \leq \frac{1}{f(r)}-\frac{1}{f(R)} .
$$

Letting $R=2 r$, we have

$$
\frac{r^{2}}{4 a r^{2}+b} \leq \frac{r^{2}}{V(2 r)} \leq \frac{r^{2}}{V(2 r)-V(r)} \leq \frac{1}{f(r)}-\frac{1}{f(2 r)} .
$$

If $f(r)$ is bounded above, then, since $f^{\prime}(r) \geq 0$, the above inequality implies that

$$
0<\frac{1}{8 a} \leq \frac{r^{2}}{4 a r^{2}+b} \leq \frac{1}{f(r)}-\frac{1}{f(2 r)} \rightarrow 0 \quad(\text { as } r \rightarrow \infty),
$$


which is a contradiction. If $f(r)$ tends to the infinity as $r \rightarrow \infty$, then we also have

$$
0<\frac{1}{8 a} \leq \frac{r^{2}}{4 a r^{2}+b} \leq \frac{1}{f(r)}-\frac{1}{f(2 r)} \rightarrow 0 \quad(\text { as } r \rightarrow \infty),
$$

which is a contradiction, too. Therefore we have $f(r) \equiv 0$, that is, $\nabla_{N} N \equiv 0$ on $L$. This fact together with Proposition 1 implies that $L$ is totally geodesic.

If $\operatorname{gr}(\mathcal{F}) \leq 2$, then, by the above argument, $\mathcal{F}$ is totally geodesic and $\nabla_{N} N \equiv 0$ on $M$. Thus $(M, g)$ is locally a Riemannian product of a leaf of $\mathcal{F}$ and an orbit of $N$. This completes the proof.

Proof of Theorem 2. Fix $x \in L$ and set $B(r)=\left\{y \in L \mid d_{L}(x, y) \leq r\right\}$ for $r \geq 0$. Define $V(r)=\operatorname{vol}(B(r))$ and $\phi(r)=\int_{B(r)}|\theta|^{2}$. As was explained in the proof of Theorem 1, the functions $V(r)$ and $\phi(r)$ are locally Lipschitz, and thus a.e. differentiable. Note that if $\nabla_{N} N \equiv 0$ on $L$, then, by Proposition 1 , we have $\operatorname{Ric}(N, N)+|A|^{2}+N(h) \equiv 0$. Thus, we may assume $\nabla_{N} N \neq 0$ at $x \in L$.

Since $L$ is of polynimial growth, there are a positive integer $k \in N$ and positive constants $a$ and $b$ so that $V(r) \leq a r^{k}+b$ for all $r \geq 0$. We take such $k \in N$ to be the smallest integer with this property, that, is, if $l<k$ is a positive integer, and $\alpha, \beta$ are any positive constants, then there is a monotone increasing sequence $\left\{r_{i}\right\}_{i=1}^{\infty}$ so that $r_{i} \rightarrow \infty$ as $i \rightarrow \infty$ and $\alpha r_{i}^{l}+\beta \leq V\left(r_{i}\right)$ for all $i$.

Assuming that $\inf _{x \in L}\left\{\operatorname{Ric}(N, N)(x)+|A|^{2}(x)+N(h)(x)\right\} \geq c>0$, we shall derive a contradiction. By this assumption and Proposition 1, it follows that

$$
c+|\theta|^{2} \leq \operatorname{div}_{L}\left(\nabla_{N} N\right) .
$$

Integrating this inequality over $B(r)$, we have

$$
c V(r)+\phi(r) \leq \int_{\partial B(r)}|\theta| \leq \sqrt{\int_{\partial B(r)} 1} \sqrt{\int_{\partial B(r)}|\theta|^{2}} .
$$

As $V^{\prime}(r)=\int_{\partial B(r)} 1>0$ and $\phi^{\prime}(r)=\int_{\partial B(r)}|\theta|^{2}$, we have

$$
(c V(r)+\phi(r))^{2} \leq V^{\prime}(r) \phi^{\prime}(r) \leq V^{\prime}(r)\left(c V^{\prime}(r)+\phi^{\prime}(r)\right) .
$$

A similar argument as in the proof of Theorem 1 shows that

$$
\frac{(R-r)^{2}}{V(R)-V(r)} \leq \frac{1}{c V(r)+\phi(r)}-\frac{1}{c V(R)+\phi(R)} \quad(0<r<R) .
$$

Thus, by taking $R=2 r$, we get

$$
\frac{r^{2}}{V(2 r)} \leq \frac{r^{2}}{V(2 r)-V(r)} \leq \frac{1}{c V(r)+\phi(r)}<\frac{1}{c V(r)} .
$$

As $V(r) \leq a r^{k}+b$, it follows that $V(2 r) \leq a 2^{k} r^{k}+b$. Therefore, we have

$$
c r^{2} V(r) \leq a 2^{k} r^{k}+b,
$$

which implies that

$$
V(r) \leq \alpha r^{k-2}+\beta
$$


for some positive constants $\alpha$ and $\beta$. But this contradicts the assumption on $k$. This completes the proof of Theorem 2.

As a corollary, by noticing that $h^{2} \leq n|A|^{2}$, we have

Corollary 1. Let $M, \mathcal{F}$ and $L$ be as in Theorem 2. Assume that the leaf $L$ is of constant mean curvature $h$ and $N(h) \geq 0$ on $L$. Then we have

$$
\inf _{x \in L}\left(\operatorname{Ric}(N, N)(x)+\frac{h^{2}(x)}{n}\right) \leq 0 .
$$

Note that, as is proved in Proposition 3, the condition $N(h) \geq 0$ implies the strong stability of a leaf of constant mean curvature. This corollary is related to the result of Alencar and do Carmo [AdC1], where the growth condition is a little bit stronger than ours (see also [AdC2]).

Let $\mathcal{F}$ be a constant mean curvature foliation. Then, as pointed out in Proposition 2, $N(h) \equiv 0$ or $N(h)$ never vanishes on $L$. Thus, we have the following corollaries for codimension-one constant mean curvature foliations of polynomial growth.

Corollary 2. Let $(M, g, \mathcal{F})$ be as in Theorem 2. Assume that $(M, g)$ is with nonnegative Ricci curvature and that $\mathcal{F}$ is a constant mean curvature foliation of polynomial growth. If $N(h)>0$ on $L \in \mathcal{F}$, then

$$
\inf _{x \in L} N(h)(x)=0 \quad \text { and } \quad \inf _{x \in L}|A|^{2}(x)=0 .
$$

By noticing again that $h^{2} \leq n|A|^{2}$, we have

COROLlary 3. Let $(M, g, \mathcal{F})$ be as in Corollary 2. If $N(h) \geq 0$ on a leaf $L \in \mathcal{F}$, then $h=0$, that is, $L$ is a minimal hypersurface.

In [M], Miranda proved that

$$
\lim _{r \rightarrow \infty} \frac{\int_{B(r)}|A|^{2}}{r^{n-2}}<\infty
$$

for a minimal graph $f: R^{n} \rightarrow R^{n+1}$ (see also [dCP]). Theorem 3 is a foliated version of this result, because the growth of a minimal graph $f: R^{n} \rightarrow R^{n+1}$ is known to be $n$ (see [GT]). However, the growth of leaves of minimal foliations of $R^{n+1}$ is not known, in general.

Proof of TheOREM 3. It follows from Proposition 1 and the assumption that

$$
|A|^{2}+|\theta|^{2}=\operatorname{div}_{L}\left(\nabla_{N} N\right) \text {. }
$$

If $|\theta| \equiv 0$ on $L$, then $|A| \equiv 0$ on $L$ and there is nothing to prove. Thus we may assume that $\nabla_{N} N \neq 0$ at $x \in L$. Set $B(r)=\left\{y \in L \mid d_{L}(x, y) \leq r\right\}, V(r)=\operatorname{vol}(B(r))$, $\psi(r)=\int_{B(r)}|A|^{2}$ and $\phi(r)=\int_{B(r)}|\theta|^{2}$. As was explained in the proof of Theorem 1, the 
functions $V(r), \psi(r)$ and $\phi(r)$ are locally Lipschitz, and thus, a.e. differentiable. Integrating the formula $(*)$ over $B(r)$ and proceed as in the proofs of Theorems 1 and 2, we get

$$
(\psi(r)+\phi(r))^{2} \leq \int_{\partial B(r)}|\theta| \leq V^{\prime}(r) \phi^{\prime}(r) \leq V^{\prime}(r)\left(\psi^{\prime}(r)+\phi^{\prime}(r)\right),
$$

from which it follows that, for $0<r<R$,

$$
\frac{(R-r)^{2}}{V(R)-V(r)} \leq \frac{1}{\psi(r)+\phi(r)}-\frac{1}{\psi(R)+\phi(R)} .
$$

Thus, by taking $R=2 r$, we get

$$
\frac{r^{2}}{V(2 r)} \leq \frac{r^{2}}{V(2 r)-V(r)} \leq \frac{1}{\psi(r)+\phi(r)} \leq \frac{1}{\psi(r)} .
$$

Therefore, by the growth condition $V(r) \leq a r^{k}+b$, we have

$$
\frac{\int_{B(r)}|A|^{2}}{r^{k-2}}=\frac{r^{2} \psi(r)}{r^{k}} \leq \frac{a 2^{k} r^{k}+b}{r^{k}}<\infty .
$$

This completes the proof of Theorem 3.

Finally, we mention a generalization of Theorem 3 . As the proof proceeds entirely in the same way as that for Theorem 3, we omit it.

THEOREM 4. Let $\mathcal{F}$ be a codimension-one minimal foliation of a complete Riemannian manifold $(M, g)$ with non-negative Ricci curvature. If the growth of $L \in \mathcal{F}$ is at most $k \in N$, then

$$
\limsup _{r \rightarrow \infty} \frac{\int_{B(r)}|A|^{2}}{r^{k-2}}<\infty .
$$

4. A concluding remark. In this section, we give a simple proof of the following result of Meeks.

THEOREM M1 (Meeks [Me]). Suppose that $\mathcal{F}$ is a $C^{2}$-foliation of $R^{3}$ such that each leaf of $\mathcal{F}$ is a surface of constant mean curvature. Then $\mathcal{F}$ consists entirely of parallel planes.

In $[\mathrm{Me}]$, the main part of the proof is showing the simply-connectedness of the leaves. Then, by Theorem 1 in [Me] (see Theorem M2 below), we obtain the desired conclusion. To show that each leaf is simply connected, he used rather complicated results and arguments. Here, we show the simply-connectedness of the leaves by an easy and a standard argument in foliation theory. For the sake of convenience, we give a complete proof of the above theorem, though the first part of the proof is similar to the one by Meeks.

ProOf. Let $\mathcal{F}$ be a constant mean curvature foliation of $R^{3}$. By Proposition 3 and a result of Silveira [Sil], $L$ is a plane if $N(h) \geq 0$. Thus, the set $W=\left\{x \in R^{3} \mid N(h) \geq 0\right\}$ is a union of parallel planes in $R^{3}$. 
Let $D$ be the set of points $x$ with $N(h)(x)<0$. By considering each connected component if necessary, we may assume that the set $D$ is diffeomorphic to $R^{3}$, since $D=R^{3} \backslash W$. By Proposition 2, the vector field $N / N(h)$ preserves $\left.\mathcal{F}\right|_{D}$. It is known that if $K \subset D$ is compact, then, for sufficiently small $\varepsilon>0$, there is a flow $\phi_{t}, t \in(-\varepsilon, \varepsilon)$, generating $N / N(h)$ on a neighborhood of $K$. By this fact, if $a(s), s \in[0,1]$, is a curve orthogonal to $\left.\mathcal{F}\right|_{D}$ and, $b(t)$, $t \in[0,1]$, is a curve on a leaf $\left.L \in \mathcal{F}\right|_{D}$ with $a(0)=b(0)$, then there is a continuous map $f:[0,1] \times[0,1] \rightarrow D$ so that $f(0, t)=b(t)$ for $t \in[0,1], f(s, 0)=a(t)$ for $s \in[0,1]$, $\left.\{f(s, t) \mid s \in[0,1]\} \perp \mathcal{F}\right|_{D}$ and $\{f(s, t) \mid t \in[0,1]\}$ is contained in a leaf of $\left.\mathcal{F}\right|_{D}$. Thus, a standard argument in foliation theory (cf. Kashiwabara [K]) implies that the lift of $\left.\mathcal{F}\right|_{D}$ to the universal covering space of $D$ is a product foliation. Since $D$ is simply connected, $\left.\mathcal{F}\right|_{D}$ itself is a product foliation. Therefore, $\left.\mathcal{F}\right|_{D} \equiv L \times R$ for some leaf $\left.L \in \mathcal{F}\right|_{D}$. As $D$ is simply connected, $L$ is also simply connected. Finally, as the mean curvature function $h$ of $\mathcal{F}$ satisfies $d h \neq 0$ on $D$, each connected component of $h^{-1}(a)(a \in R)$ is a proper hypersurface. This means that each leaf $L$ is proper in $R^{3}$.

Therefore, in order to complete the proof, we have only to use the following Meeks' result in $[\mathrm{Me}]$.

THEOREM M2 (Meeks [Me]). If $M$ is a properly embedded surface in $R^{3}$ with nonzero constant mean curvature, then $M$ is not homeomorphic to a closed surface with a single point removed.

\section{REFERENCES}

[AdC1] H. AlenCAR AND M. Do CARMo, Hypersurfaces of constant mean survature with finite index and volume of polynimial growth, Arch. Math. 60 (1993), 489-493.

[AdC2] H. Alencar AND M. Do CARMo, Erratum to "Hypersurfaces of constant mean curvature with finite index and volume of polynimial growth", Arch. Math. 65 (1995), 271-272.

[BdC] J. L. BARBOSA AND M. DO CARMO, Stability of hypersurfaces with constant mean curvature, Math. Z. 185 (1984), 339-353.

[BdCE] J. L. BARbosA, M. Do CARMo AND J. EschenburG, Stability of hypersurfaces of constant mean curvature in Riemannian manifolds, Math. Z. 197 (1988), 123-138.

[BKO] J. L. BARbosa, K. Kenmotsu AND G. OshikiRi, Foliations by hypersurfaces with constant mean curvature, Math. Z. 207 (1991), 97-108.

[BoDG] E. Bombieri, E. DeGiorgi And E. Guisti, Minimal cones and the Bernstein problem, Invent. Math. 7 (1969), 243-268

[CY] S. Y. CHENG AND S. T. YAU, Differential equations on Riemannian manifolds and their geometric applications, Comm. Pure Appl. Math. XXVII (1975), 333-354.

[dCP] M. Do CARMo AND C. K. PENG, Stable complete minimal hypersurfaces, Differential geometry and differential equations, Proceedings of the 1980 Beijing Symposium, Vol. 3 (Beijing, 1980), 1349-1358, Science Press, Beijing, 1982.

[GT] D. Gilbarg And N. Trudinger, Elliptic Partial Differential Equations of Second Order, Springer, Berlin, 1977.

[K] S. Kashiwabara, The decomposition of a differentiable manifold and its applications, Tôhoku Math. J. 11 (1959), 43-53.

[L] H. B. LAwSON, JR., Lectures on minimal submanifolds Vol. I, Publish or Perish, Inc., Berkeley, 1980.

[Me] W. MEEKS, The topology and geometry of embedded surfaces of constant mean curvature, J. Differential Geom. 27 (1988), 539-552. 
[Mi] M. MiRAndA, Una maggiorazione integrale per le curvature delle ipersuperfici minimali, Rend. Sem. Mat. Univ. Padova 38 (1967), 91-107.

[O1] G. OsHIKIRI, A remark on minimal foliations, Tôhoku Math. J. 33 (1981), 133-137.

[O2] G. OSHIKIRI, Jacobi fields and the stability of leaves of codimension-one minimal foliations, Tôhoku Math. J. 34 (1982), 417-424.

[O3] G. OSHIKIRI, Totally geodesic foliations and Killing fields, Tôhoku Math. J. 35 (1983), 387-392.

[Ra] A. RANJAN, Structural equations and an integral formula for foliated manifolds, Geom. Dedicata 20 (1986), 85-91.

[Rh] B. REINHART, Differential Geometry of Foliations, Springer, Berlin, 1983.

[Sil] A. SILVEIRA, Stable surfaces of constant mean curvature, Doctoral thesis, IMPA, Rio de Janeiro, Brasil, 1986.

[Sol] B. SOLOMON, On foliations of $R^{n+1}$ by minimal hypersurfaces, Comment. Math. Helv. 61 (1986), 67-83.

[Td] P. TONDEUR, Geometry of Foliations, Birkhäuser, Basel, 1997.

FACULTY OF EDUCATION

IWATE UNIVERSITY

18-33, UEDA 3-CHOME, MORIOKA 020-8550

JAPAN

e-mail address : oshikiri@iwate-u.ac.jp 